\title{
Skyrmionic interconnect device
}

\author{
Runze Chen $\odot,{ }^{1, *}$ Yu Li $\odot,{ }^{1}$ Vasilis F. Pavlidis $\odot,{ }^{2}$ and Christoforos Moutafis $\odot{ }^{1, \dagger}$ \\ ${ }^{1}$ Nano Engineering and Spintronic Technologies (NEST) Group, University of Manchester, Manchester M13 9PL, United Kingdom \\ ${ }^{2}$ Advanced Processor Technologies (APT) Group, University of Manchester, Manchester M13 9PL, United Kingdom
}

(Received 30 June 2020; accepted 30 October 2020; published 3 December 2020)

\begin{abstract}
The topological properties of skyrmionic quasiparticles such as magnetic skyrmions and skyrmioniums enable their applications in future low-power, ultradense nanocomputing and neuromorphic systems. We propose here an all-magnetic skyrmionic interconnect, which has so far been the "missing component." This interconnect will be of relevance to future spintronic circuits as well as quantum computing where nanofabricated interconnects need to operate at cryogenic temperatures. Here, we propose a nanoscale skyrmionic interconnect device that exploits topological selectivity to achieve signal multiplexing, thereby reducing the number of interconnects by $50 \%$ with no additional overhead for control signals. We utilize systematic micromagnetic calculations to evaluate operational parameters from ideal conditions $(0 \mathrm{~K})$ to room temperature in a tailor-designed magnetic multilayer heterostructure in order to demonstrate the potential of a skyrmion-based nanoscale spintronics interconnect. Finally, we introduce pipelining, which is achieved with strategically etched notches, in order to achieve a superior throughput in multiplexing and demultiplexing signals. Our proposal widens the possibilities for future spintronics-based nanocomputing.
\end{abstract}

DOI: 10.1103/PhysRevResearch.2.043312

\section{INTRODUCTION}

Although transistors have traditionally been the workhorse of integrated systems, interconnects have become the bottleneck in multibillion-transistor integrated systems [1]. The power spent for transferring data among integrated components or chiplets has steadily risen in the past few years, exceeding often the energy spent for computation [2-4]. This situation has led the industry to pursue solutions that exploit physical proximity to reduce the interconnect length both on chip and between components [5-7]. Alternatively, neuromorphic computing, which utilizes brain-inspired principles to design hardware with superior power efficiency [8], requires massive interconnect density to support modern deep neural networks [9]. Moreover, future large-scale quantum computers with millions of qubits also pose a challenge in terms of the effective interconnect between subkelvin qubits and room-temperature measurements $[10,11]$.

Recently, skyrmion-based neural networks have been proposed to address the issues of energy efficiency, information volatility, and synapse plasticity by combining skyrmionic devices with complementary metal oxide semiconductor (CMOS) technologies in highly energy-efficient hybrid neural networks [8,12-14]. However, there is no efficient solution

\footnotetext{
*runze.chen@manchester.ac.uk

†christoforos.moutafis@manchester.ac.uk
}

Published by the American Physical Society under the terms of the Creative Commons Attribution 4.0 International license. Further distribution of this work must maintain attribution to the author(s) and the published article's title, journal citation, and DOI. to provide the high number of interconnections demanded in neural networks irrespective of the underlying technology. Recognizing this demand and the entailed routing complexity and overhead in energy, a novel skyrmionic interconnect is in high demand and has not yet been explored so far. The skyrmionic interconnect may also be of relevance to future large-scale quantum computers operating at cryogenic temperatures as low as $0.01 \mathrm{~K}$, where the temperature gap between qubits and control circuits remains [10,15].

Magnetic skyrmions are topologically protected spin textures with particlelike properties [16]. Chiral skyrmions have been demonstrated in both bulk noncentrosymmetric chiral magnets and magnetic multilayer (MML) thin films with the Dzyaloshinskii-Moriya interaction (DMI) present [17]. Skyrmion-based devices can be used in hybrid solutions compatible with CMOS technologies due to their robustness, nanoscale size, and nonvolatility [16]. Skyrmions can be nucleated, manipulated, and deleted via various external stimuli $[18,19]$, which sheds light upon their usage in power-efficient nanocomputing applications. The enhanced stability and robustness of skyrmions can be seen as a result of their topology [16] and energy contributions [20]. Skyrmionic spin textures carry a nonzero, integer value topological charge, also called the skyrmion number [21], which counts how many times the vector field configuration wraps around a unit sphere. It is defined as

$$
N=\frac{1}{4 \pi} \int \mathbf{m} \cdot\left(\partial_{x} \mathbf{m} \times \partial_{y} \mathbf{m}\right) d x d y,
$$

where $N= \pm 1$ for the case of skyrmions and its sign reflects the polarity.

Skyrmions experience the skyrmion Hall effect (SkHE) under electric currents [22-24], which is effectively akin to 
the bubble skew deflection under magnetic field gradients, first reported in the 1970s [25-27]. In high-speed operation, the transverse motion of a magnetic skyrmion may result in its annihilation at the edge of the nanostructure, which leads to a drastically reduced reliable distance for skyrmion transport [28]. Many research efforts of the community have so far focused on controlling and suppressing the SkHE, such as introducing high perpendicular magnetic anisotropy at edges [29], modifying the spin Hall angle [30], introducing the synthetic antiferromagnetic (SAF) structure [31] and utilizing topologically trivial textures, such as skyrmioniums [24,3234].

The skyrmionium, a skyrmion-antiskyrmion pair, is another type of magnetic quasiparticle with a zero total topological charge, which was originally referred to as a $2 \pi$ vortex in Ref. [35]. Skyrmionium-based nanodevices were originally envisaged as potential approaches in order to overcome the SkHE [24], while on the contrary, exploiting the SkHE coupled with topological selectivity, in general, in nanodevices has so far been largely unexplored. Hitherto, skyrmionic on-site memory, nanocomputing, and neuromorphic components have increasingly been investigated by the community [36]. However, the "missing component" in skyrmionics so far has been an all-magnetic skyrmion-based interconnect. The characteristics of the skyrmionic interconnect may enable efficacious signal transfer and propagation at emerging computing paradigms, such as quantum computing where cryogenic temperatures are required [15,37].

Nanomagnets can host a plethora of topological and nontopological quasiparticles. Specifically, skyrmionic quasiparticles with various topological charges intrinsically behave differently by exhibiting different skyrmion Hall angles [38]. It is therefore tantalizing to exploit the distinct topological and nontopological signatures of skyrmionic quasiparticles for on-demand topological filtering. We propose here a way to implement such a nanoscale skyrmionic interconnect device for signal multiplexing. This skyrmionic interconnect resembles conceptually the wavelength multiplexing in silicon photonics [39] and the signal multiplexing based on wave pipelining in standard CMOS interconnects [40].

In this paper, we (i) leverage the SkHE and (ii) propose using both topologically nontrivial skyrmions and trivial skyrmioniums as information carriers, while also (iii) exploring the potential future use of topological particles with more complex spin textures. Rather than using skyrmions or skyrmioniums individually as information carriers [16,41], we propose to encode two sequences of information by utilizing both along a racetrack-type device. The combination of different types of skyrmionic textures as information carriers effectively enables signal multiplexing. The information carriers of the proposed skyrmionic interconnect inherently choose the right output via exploiting the SkHE for topological selectivity, circumventing the need for a control signal that is causing the overhead at the output side. Therefore, in our proposed device, we save signal routing by $50 \%$. We perform micromagnetic simulations to demonstrate the feasibility of the device and its operational window, as well as to demonstrate an example of a multiplexing operation. We compute the operational window of the proposed skyrmionic interconnect by carefully tuning the device structure parameters.
Moreover, we evaluate the thermal behavior of the device and enhance the thermal stability by introducing a tailored MML structure (e.g., Ref. [42]). We then strategically etch notches in the racetrack to guarantee the precise detection of both information signals. Furthermore, we introduce pipelining in the skyrmionic interconnect, which can significantly increase the signal throughput. Finally, we envisage a way to expand the capacity for multiplexing and demultiplexing signals of the proposed device, by introducing a double-antiskyrmionskyrmion pair (which we call here an antiskyrmionite) as a third type of information carrier. Our results suggest that skyrmionic quasiparticles with various topological charges can be suitable for novel nanoscale spintronics interconnect devices.

\section{RESULTS AND DISCUSSION}

This section describes the design of the proposed skyrmionic interconnect device for topological multiplexing and its evaluation via micromagnetic simulations. By carefully investigating the parameters of our proposed skyrmionic interconnect, an operational window is given for the design of the device structure. We then discuss thermal effects and a tailored MML structure for enhanced thermal stability. We introduce pipelining in the skyrmionic interconnect device by strategically etching notches to obtain a higher throughput in multiplexing and demultiplexing signals. Finally, we propose an approach to improve the performance of our proposed skyrmionic interconnect device by introducing a third type of topological information carrier.

\section{A. Nanoscale skyrmionic interconnect device for topological multiplexing}

In telecommunications and computer networks, multiplexing is a method by which multiple analog or digital signals are combined into one stream over a shared medium $[43,44]$. The proposed skyrmionic interconnect is schematically shown in Fig. 1(a), which consists of two inputs, two outputs, and a shared nanotrack. The navy blue region represents the ferromagnetic (FM) nanotrack and the yellow region is the heavy metal (HM).

As for the skyrmion-based communication, we envisage two separate signal sources multiplexed toward two separate destinations downstream on our modified racetrack. Here binary information [e.g., "1" ("0")] of the first signal is encoded by the presence (absence) of a skyrmion, while the second signal is represented in the same way but utilizes a different information carrier, which is a skyrmionium [21,41]. The geometry of our proposed device is tailor designed to intrinsically choose the correct output, exploiting the topological charge of each carrier via the SkHE, and can circumvent the need for a control signal at the receiver side. Therefore, in the proposed skyrmionic device we reduce the number of interconnect by $50 \%$ with no additional overhead for a control signal.

Magnetic skyrmions and skyrmioniums [Figs. 1(b) and 1(c)] can be nucleated by an electrical writing method reported in Refs. [33,41,45]. A spin current is injected perpendicularly into the FM layer from the HM layer because 
(a)

Signal 1

The presence of a skyrmion encodes logic " "1"

The absence of a skyrmion encodes logic "O"

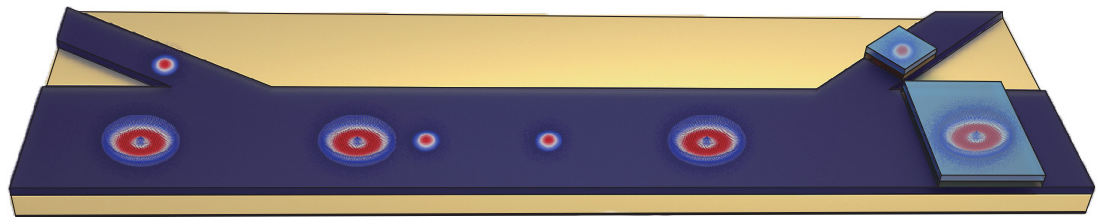

Signal 2

The presence of a skyrmionium encodes logic " 1 "

The absence of a skyrmionium encodes logic "0" (b)

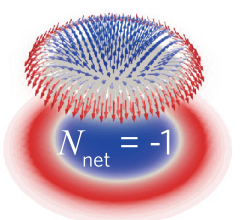

(c)

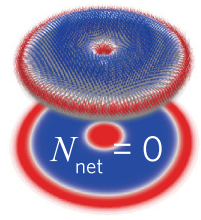

(d)

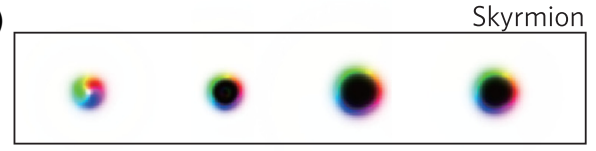

(e)

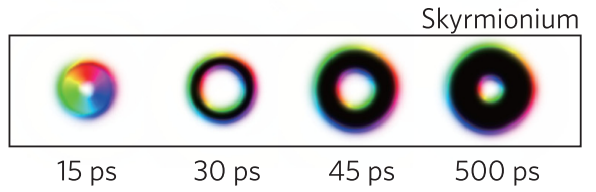

FIG. 1. The proposed skyrmionic interconnect device. (a) Schematic drawing of the proposed skyrmionic topological multiplexing device (skyrmionic interconnect). Spin texture of (b) a Néel skyrmion and (c) a skyrmionium. Arrows point in the direction of the magnetization, and $m_{z}$ is color coded from blue $(-1)$ to red $(+1)$. The nucleation process of (d) a skyrmion and (e) a skyrmionium by a writing current pulse of 15 ps duration (full width at half maximum) with $j_{\max }=2 \times 10^{13} \mathrm{~A} \mathrm{~m}^{-2}$ injected from a 40-nm-diam round disk and a 60 -nm-diam ring, respectively.

of the spin Hall effect (SHE). The spins are oriented perpendicular to the vector of the plane and the direction of charge current. After the spin current (injected via a 40-nm-diam disk and a 60-nm-diam ring, respectively) has induced a skyrmion and skyrmionium-shaped excitation, the quasiparticles relax and equilibrate in a time period of $\sim 500 \mathrm{ps}$ as shown in Figs. 1(d) and 1(e), which is in good agreement with reported results [24,33,41]. After nucleation, the skyrmions and skyrmioniums propagate toward the output branches along the $x$ direction via the electric current, as shown in Fig. 1(a). The motion of skyrmions and skyrmioniums can be controlled by a current in plane (CIP) flowing through the FM nanotrack or by a current perpendicular to the plane (CPP) [46]. CPP works better for skyrmions because a skyrmion can receive a larger Slonczewski in-plane torque than the fieldlike out-of-plane torque generated by an equal current density [45]. Skyrmions obtain higher velocities under CPP for a given current density. Meanwhile, it was found that the driving current with the CPP geometry can result in a better mobility of the skyrmionium than CIP [24]. Therefore, in this paper, we consider the case of CPP. The information is then detected by two magnetic tunnel junctions (MTJs) $[47,48]$ placed at the output branches in Fig. 1(a). Detecting the presence or absence of a skyrmion and skyrmionium can be interpreted as an information bit with value 1 or 0 , respectively.

The functionality of our proposed skyrmionic interconnect is enabled by the design of a shape that exploits the different skyrmion Hall angles of a skyrmion and a skyrmionium. Micromagnetic simulations in Fig. 2 depict the motion of a Néel skyrmion and a skyrmionium driven by an electric current $j_{e}$ (white) applied in the HM layer. The polarization of the spin current perpendicularly injected (black) into the ferromagnetic layer is in the $+y$ direction (sky blue). The skyrmion propagates first toward the upper edge and then along the direction of the driving current $(+x)$, while the skyrmionium propagates strictly along the $+x$ direction. Note that the geometry of the track will affect the device fidelity. The narrowest track geometry is determined by the size of the single skyrmion and skyrmionium. However, when the track is too wide, the transverse moving ( $t_{0}$ to $t_{2}$ in Fig. 2) of skyrmions, originating from the SkHE, will be parasitic to device performance by severely affecting the synchronization of the skyrmion and skyrmionium for signal detection. Concerning the length of the track, the shortest length should be greater than the longitudinal displacement of the skyrmion during the transverse moving process.

The topological multiplexing, which from an information system perspective is equivalent to signal multiplexing, means in practice that, after propagating in the $x$ direction along the nanotrack and reaching the output branches, the skyrmionium will move straight and forward to the lower branch (skyrmionium branch). On the other hand, the skyrmion's trajectory is diverted into the upper branch (skyrmion branch). Therefore, the multiplexing of two signals by utilizing topological and nontopological types of quasiparticles is numerically demonstrated. We then carefully investigate the operating parameters of the topological multiplexing functionality of our proposed skyrmionic device by modifying the device structure. As shown in Fig. 3(a), the width and angle of the skyrmion branch are gradually tuned within a large variation range. The topological multiplexing results are evaluated and labeled,

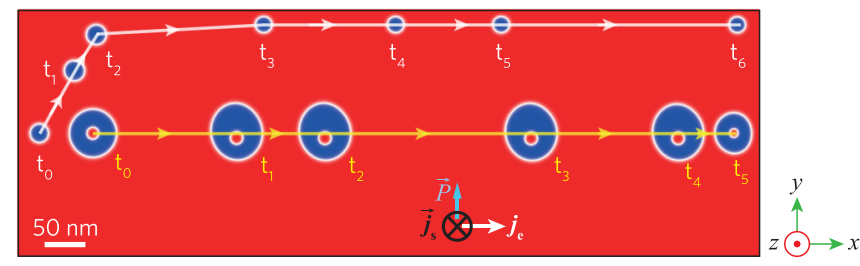

FIG. 2. Schematic of the skyrmion Hall effect for a skyrmion and a skyrmionium. Micromagnetic simulation results of the motion of a Néel skyrmion and a skyrmionium driven by a current perpendicular to the plane. The skyrmion gains a transverse velocity $\mathbf{v}_{y}$ with respect to the direction of the charge current $(+x)$ while the topologically trivial skyrmionium strictly moves along the $+x$ direction. 
(a)
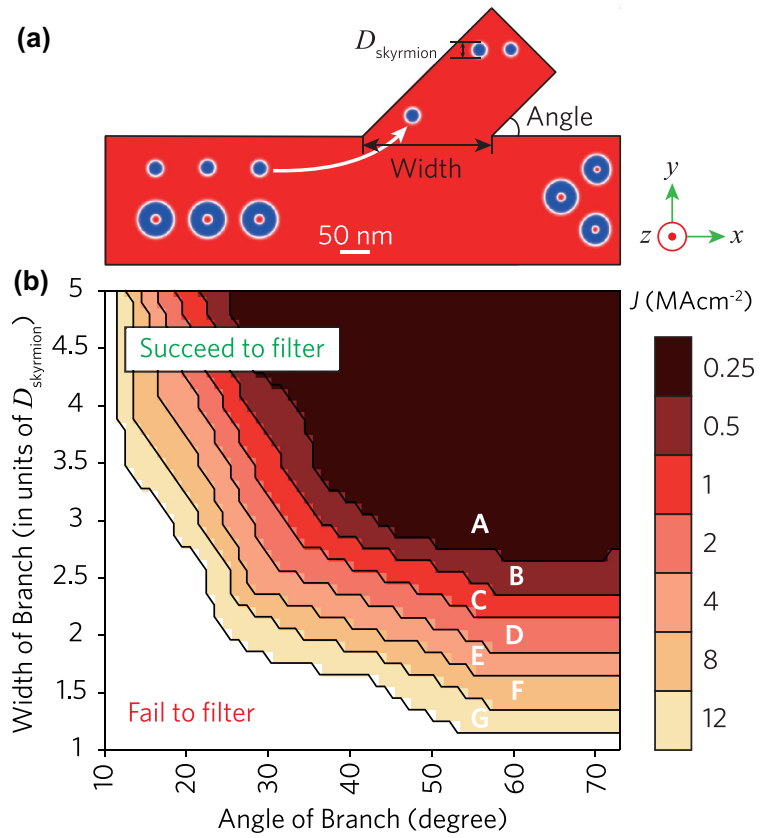

FIG. 3. Operational window of the proposed skyrmionic interconnect device. (a) Schematic illustration for tuning the width and angle of skyrmion branch in the proposed device. (b) The threshold or minimum current required for filtering, with respect to different device parameters (the angle and the width of the skyrmion branch). The color coding represents densities of the threshold current for different device parameters. The color of an enclosed region denotes the threshold current required for filtering in the interconnect device. For example, with the current amplitude of $1 \mathrm{MA} \mathrm{cm}^{-2}$, the device succeeds in filtering within regions $\mathrm{A}, \mathrm{B}$, and $\mathrm{C}$.

according to whether the skyrmion propagates successfully into the upper skyrmion branch. The operational window of our proposed skyrmionic interconnect structure with respect to the angle and the width of the skyrmion branch is shown in Fig. 3(b). The larger amplitude of the driving current leads to a larger region for successful operations in terms of the angle and width of the skyrmion branch. As shown in Fig. 3(b), the color of an enclosed region denotes the threshold current required for filtering in the interconnect device. With the current amplitude of $0.25 \mathrm{MA} \mathrm{cm}^{-2}$, the device succeeds in filtering with parameters within region A. However, with the current amplitude of $12 \mathrm{MA} \mathrm{cm}^{-2}$, the device succeeds in filtering within all the regions (A, B, C, D, E, F, and G). Note that the angle and width for the skyrmion branch for region $\mathrm{A}$ make the most reliable structural parameters of the interconnect, because within region A successful multiplexing is obtained with all current densities utilized in simulations $(0.25$ to $12 \mathrm{MA} \mathrm{cm}^{-2}$ ).

The energy consumption for multiplexing two information bits is given by

$$
E=\rho t l w J^{2} T_{\text {mux }},
$$

where $\rho$ is the resistivity of the Pt thin film [49], $t$ and $w$ are the thickness and width of the bottom HM layers, $l$ is the length of the racetrack, $J$ is the amplitude of current density, and $T_{\operatorname{mux}}$ is the duration of the pulses applied on the device to multiplex and demultiplex two information bits (50 ns for (a)

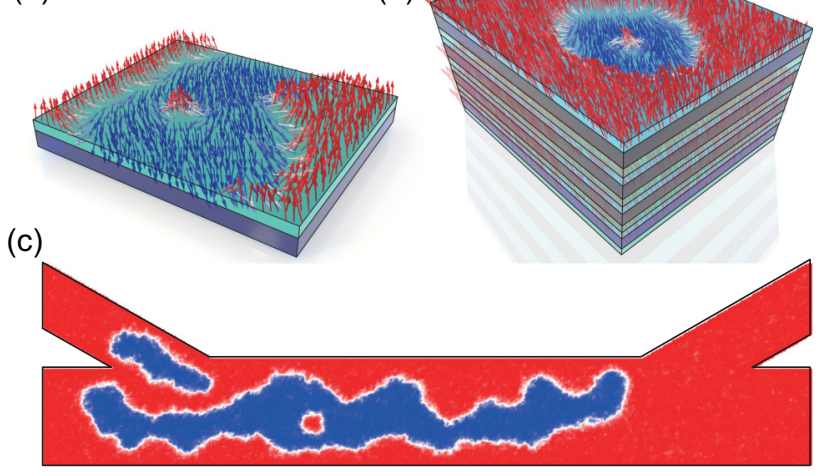

(d)

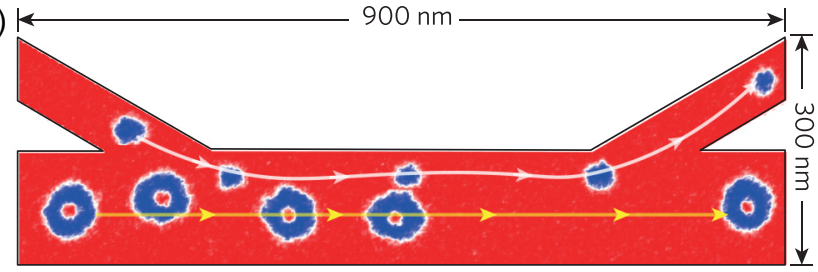

FIG. 4. The multiplexing functionality of the proposed skyrmionic interconnect at room temperature. Schematic illustration of (a) a single layer and (b) MML structure. Micromagnetic simulations of the proposed device at room temperature with (c) single-layer structure and (d) MML structure composed of several repetitions of the $[\mathrm{HM} / \mathrm{FM} / \mathrm{HM}]$ trilayers, pointing to the necessity of the MML structure for robust room-temperature functionality.

$J=1 \mathrm{MA} \mathrm{cm}^{-2}$ in a $1-\mu m$ racetrack). The energy consumption $E$ of the proposed skyrmionic interconnect during the multiplexing and demultiplexing process is estimated to be $\sim 0.7 \mathrm{fJ} / \mu \mathrm{m}$ per information bit from Eq. (2) by using the simulation parameters in Appendix, which is comparable with the energy required for a local interconnect at $180 \mathrm{~nm}$ CMOS technology (based on typical technology models [50]).

Based on the fact that realistic devices, depending on the application domain, may need to operate at room temperature, the robustness of our proposed skyrmionic interconnect at room temperature is essential. In order to simulate the skyrmionic multiplexing at room temperature $(300 \mathrm{~K})$, we introduce the finite-temperature effects in MUMAX $^{3}$ through a randomly fluctuating thermal field [51]:

$$
\vec{B}_{\text {therm }}=\vec{\eta}(\text { step }) \sqrt{\frac{2 \alpha k_{\mathrm{B}} T}{\mu_{0} M_{\mathrm{sat}} \gamma_{\mathrm{LL}} V \Delta t}},
$$

where $\alpha$ is the damping parameter, $k_{\mathrm{B}}$ is the Boltzmann constant, $T$ is the temperature, $M_{\text {sat }}$ is the saturation magnetization, $\gamma_{\mathrm{LL}}$ is the gyromagnetic ratio, $V$ is the cell volume, $\Delta t$ is the time step, and $\vec{\eta}$ (step) is a randomly oriented normal vector whose value is changed after each time step.

The simulation result in Fig. 4(c) shows that thermal effects at room temperature may destroy the functionality of the skyrmionic interconnect with a simple FM|HM structure [Fig. 4(a)], because of the expansion and instability of the room-temperature skyrmion and skyrmionium, for the given material parameters and structure. In order to stabi- 
lize room-temperature skyrmions and skyrmioniums and have a working skyrmionic interconnect that is technologically relevant, we propose a tailored MML structure inspired by recent experimental demonstrations (e.g., Ref. [42]). The basic unit of the structure is a $\left[\mathrm{HM}_{1}|\mathrm{FM}| \mathrm{HM}_{2}\right]$ sandwiched trilayer [Fig. 4(b)]. The enhanced thermal stability of skyrmions and skyrmioniums in Fig. 4(d) is enabled by (i) the enhanced DMI from the asymmetric interfaces $\left[\mathrm{HM}_{1} \mid \mathrm{FM}\right]$ and $\left[\mathrm{FM} \mid \mathrm{HM}_{2}\right]$ heterostructures [42], and (ii) adding magnetic volume by additional repeated stacks of $\left[\mathrm{HM}_{1}|\mathrm{FM}| \mathrm{HM}_{2}\right]$ sandwiched trilayers. Micromagnetic simulations with repeat number $n=4$ for magnetic multilayers $\left[\mathrm{HM}_{1}|\mathrm{FM}| \mathrm{HM}_{2}\right]_{n}$ are shown in Fig. 4(d). Compared to the single FM|HM structure in Fig. 4(a), skyrmions and skyrmioniums in our proposed interconnect device composed of the MML structure exhibit much more stable behavior which corresponds to the single $\mathrm{FM} \mid \mathrm{HM}$ structure at $0 \mathrm{~K}$. Considering that this paper aims to provide an important concept of skyrmion-based interconnect, thermal fluctuation at finite temperature is not considered in our main results.

However, in addition to the shape distortion of information carriers, finite-temperature-induced fluctuations may also distort the path of the information carriers even if their shapes are conserved. This may also cause filtering errors of the proposed interconnect device. In this paper, we use a pass/fail criterion for the device on the filtering of the skyrmion into the proper track, as depicted in Fig. 3. When the device operates at high temperatures, the pass/fail criterion may be a less credible device fidelity measure. Therefore, further research should be undertaken, especially on the design of a more precise diagnostic for the fidelity of the device, and the stability of information carriers against thermal fluctuations, as well as the inhomogeneity in magnetic parameters. To that end, utilizing additional simulation techniques such as the Monte Carlo method [52] would be necessary.

\section{B. Pipelining for the skyrmionic interconnect}

The functionality of the skyrmionic interconnect relies on the precise detection of the absence or presence of the skyrmion or skyrmionium at the corresponding branch. Therefore, a synchronization mechanism must be provided to ensure that skyrmions and skyrmioniums arriving from different input paths are detected at the reading region simultaneously. We place two repulsive right triangle notches (with hypotenuses of 40 and $80 \mathrm{~nm}$, respectively, feasible with standard lithographically accessible length scales [53]) with nonmagnetic texture in both output branches, as shown in Fig. 5. The synchronization is achieved by these notches, because the skyrmions and skyrmioniums cannot traverse the notch until a large enough spin Hall current pulse is applied. The notch depth and the current amplitude are carefully chosen to ensure that skyrmions and skyrmioniums are immobilized with a small current $j_{e}=1 \mathrm{MA} \mathrm{cm}-2$ and cross over the notches with a larger current density $\left(j_{e}=3 \mathrm{MA} \mathrm{cm}^{-2}\right)$, as shown in Figs. 5(a) and 5(b).

Based on this geometry, we numerically propose a pipelining scheme for the skyrmionic interconnect, as depicted in Fig. 6. Notches are inserted evenly in the nanotrack between input paths and output branches, dividing the race- (a)

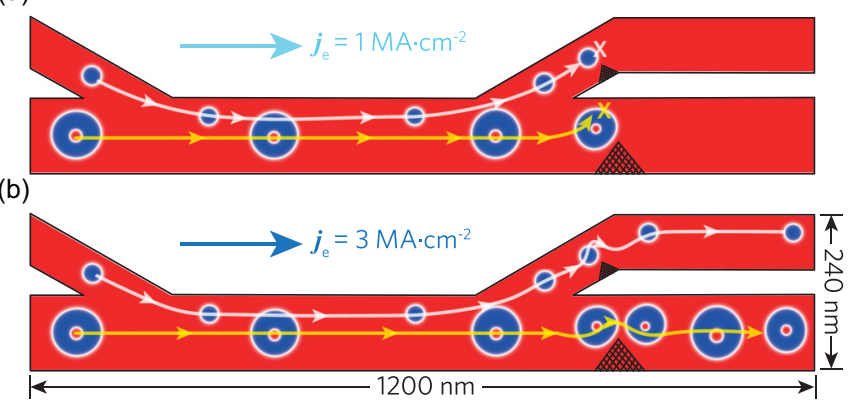

FIG. 5. Signal synchronization by introducing notches for the detection of skyrmions and skyrmioniums during the demultiplexing process. Notches are etched in the skyrmion and skyrmionium branches, respectively, forming a constriction that permits the passage of skyrmions and skyrmioniums only when an appropriate electric current is applied.

track into five regions. Each intranotch region will host zero or one skyrmion or skyrmionium during each clock cycle. The pipelined skyrmionic interconnect is powered by a series of stair-stepping electrical pulses with an amplitude of $3 \mathrm{MA} \mathrm{cm}^{-2}$ for $5 \mathrm{~ns}$ and $1 \mathrm{MA} \mathrm{cm}{ }^{-2}$ for $20 \mathrm{~ns}$. The depth of notches we employ to synchronize skyrmion and skyrmionium sequences is selected according to Ref. [45], which is experimentally feasible with industrially relevant material systems at lithographically accessible length scales.

Micromagnetic simulations in Fig. 6 show that the proposed device correctly multiplexes and demultiplexes two nine-bit messages within 12 electrical pulses. Two bitstreams (101100011 and 011101101, respectively) are set as inputs to the pipelined skyrmionic interconnect device. Within each stair-stepping electrical pulse described above, one (zero) skyrmion or skyrmionium is nucleated and shifted if the corresponding information bit to be sent is $1(0)$. The detection happens in each branch by MTJs in the last 1 ns of each stair-stepping electrical pulse and the presence (absence) of the skyrmion or skyrmionium is interpreted as $1(0)$ at the outputs. Since notches divide the racetrack into five regions, the information bit sent from the input branches will be detected after four electrical pulses.

The maximum throughput (data bandwidth capacity) of the proposed skyrmionic interconnect is given by

$$
X=\frac{C}{\tau},
$$

where $X$ is the maximum throughput of the proposed device, and $C$ is the total amount of transferred data within time period $\tau$. Assuming that the length of the racetrack is $2 \mu \mathrm{m}$, the maximum throughput of the proposed skyrmionic interconnect in Fig. 5 is calculated as 2 bits $/ 100 \mathrm{~ns}=20 \mathrm{Mbps}$ from Eq. (4). In comparison, the proposed pipelined skyrmionic interconnect in Fig. 6 demonstrates the ability to provide a significant improvement of the throughput, 2 bits $/ 25 \mathrm{~ns}=$ $80 \mathrm{Mbps}$, despite a 100-ns latency (four pluses) to produce the demultiplexed outputs. 


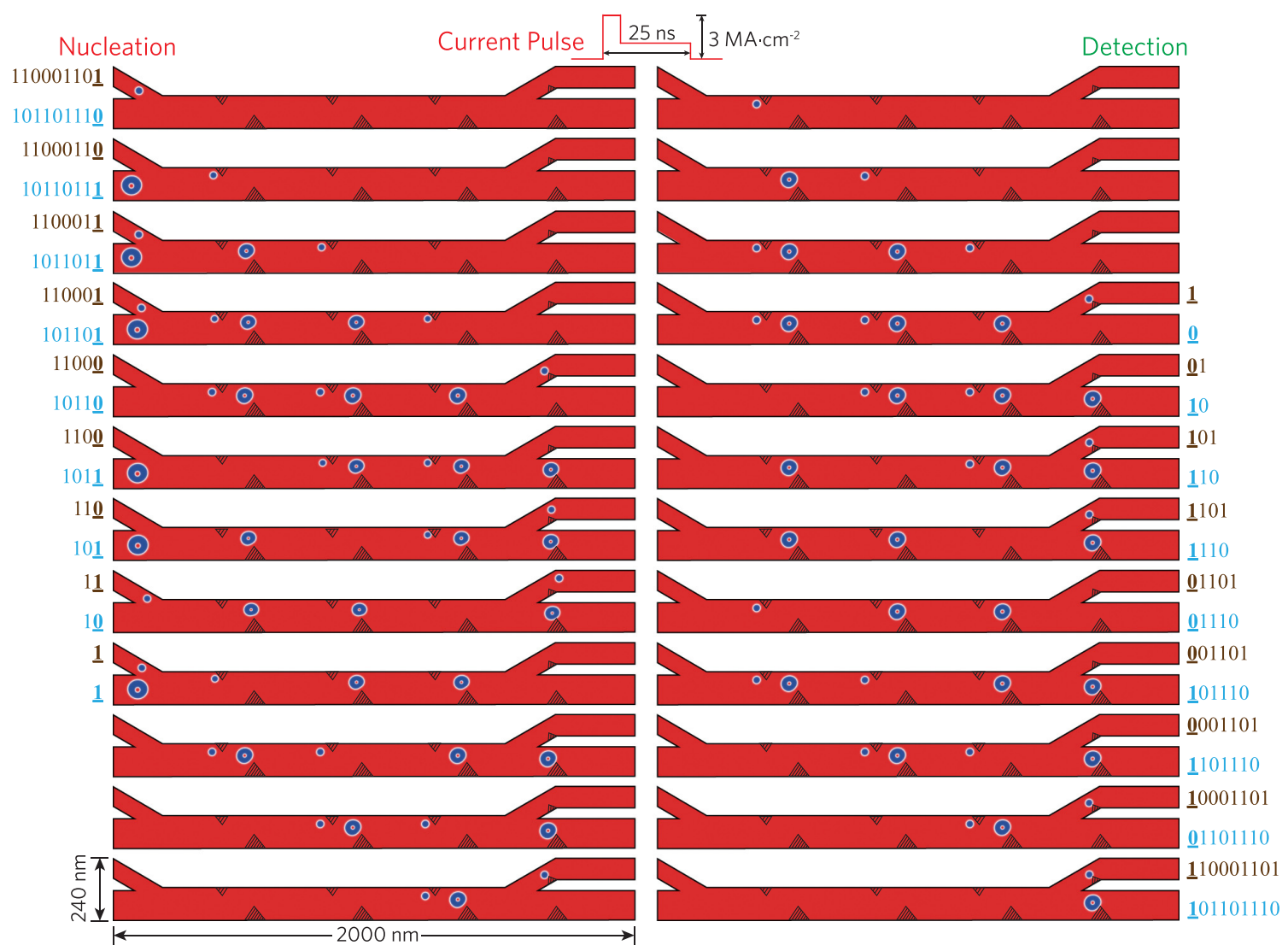

FIG. 6. Micromagnetic simulations of the pipelined skyrmionic interconnect. The racetrack is divided into five regions via strategically etched notches. The device is powered by a series of stair-stepping electrical pulses with an amplitude of $3 \mathrm{MA} \mathrm{cm}^{-2}$ for $5 \mathrm{~ns}$ and $1 \mathrm{MA} \mathrm{cm}-2$ for $20 \mathrm{~ns}$.

\section{Topological multiplexing for multiple types of information carriers}

In addition to the skyrmion and skyrmionium, it has been shown theoretically that in chiral magnets it is possible to have an infinite set of skyrmion solutions with different value and sign of topological charges [38]. Furthermore, bubbles with arbitrary topologies were experimentally investigated since the 1970s [26]. It has been numerically shown that topologically trivial bubbles, arising from topological defects in their domain wall, can also be metastable states in nanomagnets [21] which could also be relevant in the context of magnetic memories. Quite recently, stable and high-degree multiskyrmion configurations where an arbitrary number of antiskyrmions are contained within a larger skyrmion were experimentally demonstrated in liquid crystals [54]. Note that while there is potentially a plethora of topological and nontopological excitations, it is not straightforward that they will be stable or metastable quasiparticles that can be utilized as information carriers. Based on these observations, we extend our proposed skyrmionic interconnect by introducing a third type of information carrier, in order to illustrate the capability and possibility of a higher-degree skyrmionic interconnect (e.g., as shown experimentally in liquid crystals [54]). This is a complex spin structure with a total net topological charge of +1 , which acts as an effective antiskyrmion in this system. For convenience, we propose to call this quasiparticle an antiskyrmionite. In our simulation, we stabilize the antiskyrmionite by setting up an initial ansatz with the shape of a double-antiskyrmion-skyrmion pair and letting it equilibrate in situ. We also check below its stability under current during propagation. Note that we choose the antiskyrmionite as the third quasiparticle rather than a simpler, single antiskyrmion because (i) in order to stabilize metastable antiskyrmions in thin films, the sign of the DMI has to be opposite along two in-plane directions of the film. Therefore, special symmetries in the material are required (e.g., thin films with $\mathrm{C}_{2 v}$ symmetry $[55,56])$. (ii) There may exist a pair annihilation of a skyrmion and an antiskyrmion in a forced collision event in a given system (e.g., in frustrated magnets [57]).

As seen in Fig. 7(a), our micromagnetic simulations show that, under the same spin Hall current, a Néel skyrmion $\left(N_{\text {net }}=-1\right)$ tends to obtain a transverse velocity in the $+y$ direction; a skyrmionium $\left(N_{\text {net }}=0\right)$ tends to move straight forward along the $+x$ direction; and the antiskyrmionite $\left(N_{\text {net }}=+1\right)$ tends to obtain a transverse velocity in the $-y$ direction. We evaluate the functionality of the device in order to demonstrate that the proposed skyrmionic interconnect with three branches is able to filter quasiparticles with three different topologies. As shown in Fig. 7(b), the skyrmioniums $\left(N_{\text {net }}=0\right)$ propagate into the middle output branch, while Néel skyrmions $\left(N_{\text {net }}=-1\right)$ and double-antiskyrmionskyrmion pairs or antiskyrmionite quasiparticles $\left(N_{\text {net }}=+1\right)$ are respectively driven into the corresponding branches. Note 
(a)

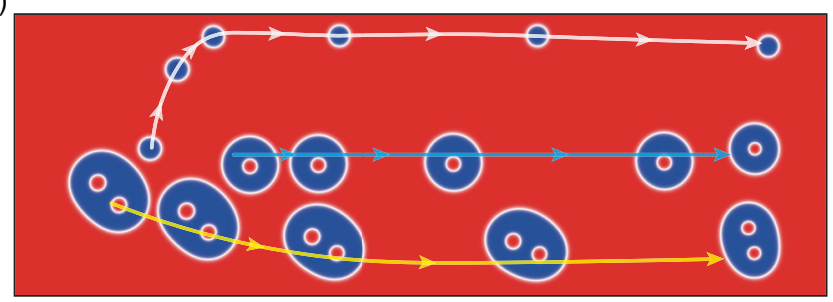

(b)

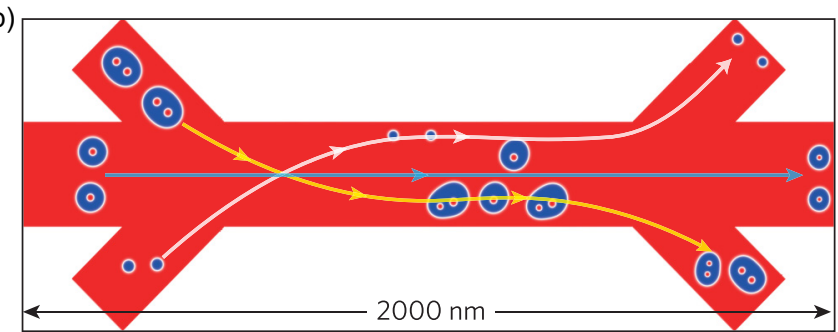

(c)

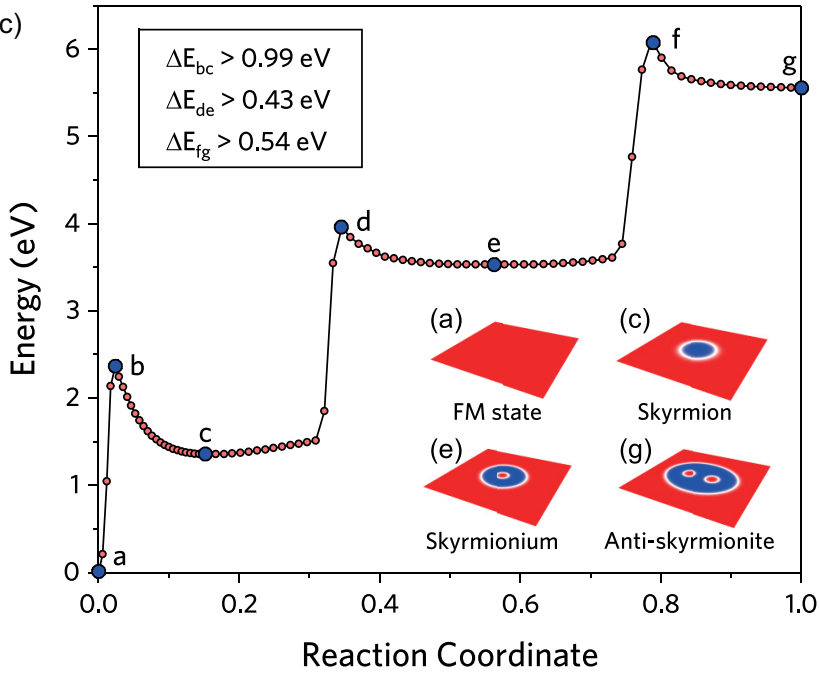

FIG. 7. Topological multiplexing for more than two types of information carriers. (a) Micromagnetic simulation results of the motion of a Néel skyrmion $\left(N_{\text {net }}=-1\right)$, a skyrmionium $\left(N_{\text {net }}=0\right)$, and an antiskyrmionite $\left(N_{\text {net }}=+1\right)$ driven by CPP. (b) Demonstration of the proposed skyrmionic interconnect for three types of information carriers. (c) Minimum energy paths of a skyrmion, a skyrmionium, and an antiskyrmionite in a nanodisk. The reaction coordinate is an order parameter that represents the relative distance between the states in the configuration space. The local minima a, c, e, and $\mathrm{g}$ correspond to the energies of the FM state, the skyrmion, the skyrmionium, and the antiskyrmionite, respectively (see the snapshots of micromagnetic simulations in the corresponding insets).

that our proposed skyrmionic interconnect can also be envisaged to utilize frustrated magnets, in order to embed skyrmionic topological quasiparticles with multiple distinct topological charge states [57,58].

In order to motivate studies on nanodevices using multiple topological and nontopological textures as quasiparticles and/or information carriers, their stability must be further investigated. We study the energy paths of the transitions between the FM state, the skyrmion, the skyrmionium, and the antiskyrmionite in a nanodisk. As shown in Fig. 7(c), minimum energy transitions between four equilibrium states are calculated via the nudged elastic band method (NEBM)
$[59,60]$. The difference between the energy values in the local minima [see points c, e, and g in Fig. 7(c)] and nearest maxima corresponding to the saddle points [see points $\mathrm{b}, \mathrm{d}$, and $f$ in Fig. 7(c)] defines the energy barrier underlying the stability of the corresponding state. From the calculation of the NEBM, we can obtain energy barriers $\Delta E_{\mathrm{bc}}>0.99 \mathrm{eV}$, $\Delta E_{\mathrm{de}}>0.43 \mathrm{eV}$, and $\Delta E_{\mathrm{fg}}>0.54 \mathrm{eV}$ for the transitions from a skyrmion to the FM state, a skyrmionium to a skyrmion, and an antiskyrmionite to a skyrmionium, respectively. Interestingly, the antiskyrmionite has a higher energy barrier than the skyrmionium. As one would also expect from topological arguments, the topologically nontrivial quasiparticles (skyrmion and antiskyrmionite) are more stable than topologically trivial textures like the skyrmionium. According to the calculations in Refs. [60-62], it is possible that quasiparticles could be unstable at room temperature, which would therefore affect the device fidelity. However, since the calculation of the energy barriers of quasiparticles is highly determined by magnetic parameters, the energy barriers of quasiparticles can be vastly enhanced by appropriately choosing material systems [62], as well as carefully designing device structures. Moreover, in some emerging systems such as frustrated magnets and liquid crystals, it is promising that multiple metastable quasiparticles, even antiskyrmions, are expected to coexist and be stable at room temperature [54,57,58]. Future work should be conducted to further investigate the stability of such textures. This would allow for a wider scope in experiments that go beyond the magnetic skyrmion, as well as for enabling richer possibilities in devices. It would be tantalizing to consider possible pathways for tuning the skyrmion-FM, skyrmion-skyrmionium, and antiskyrmioniteskyrmionium energy barriers in order to design and implement realistic topology-based spintronic nanodevices.

\section{CONCLUSIONS}

The intrinsic physics properties of skyrmionic quasiparticles with different topological charges enable skyrmionic devices to be utilized in high-degree information storage and processing. In this work, we propose an element that has been missing so far from the emerging field of skyrmion electronics. We describe computationally a nanoscale skyrmionic interconnect device for topological multiplexing, where two sequences of propagating skyrmions and skyrmioniums are encoded as specific patterns that represent two information signals. The patterns of skyrmions $\left(N_{\text {net }}=-1\right)$ and skyrmioniums $\left(N_{\text {net }}=0\right)$ are nucleated, driven and detected within the FM nanotrack. The information carriers of the proposed skyrmionic interconnect inherently choose the right output via exploiting the SkHE for topological selectivity, circumventing the need for a control signal at the output side. Therefore, in the proposed skyrmionic device we reduce the number of interconnect wiring by $50 \%$ with no additional overhead for control. In order to enhance the thermal stability, a tailored MML structure is introduced so that the skyrmionic interconnect device performs robustly at room temperature. Based on the design of appropriately etched notches, we propose and evaluate a pipelined skyrmionic interconnect to obtain a superior signal throughput. Furthermore, we test and verify the possibility of topological multiplexing for more than two 
types of information carriers by introducing an antiskyrmionite quasiparticle $\left(N_{\text {net }}=+1\right)$. As future research, a more fine-tuned diagnostic for the fidelity of the device beyond a pass/fail criterion on the filtering should be considered, particularly at higher temperatures. The results here show the possibility for skyrmionic interconnects as the "missing component" in skyrmionics that complements memory and nanocomputing devices, and also shed light on skyrmionic devices with multiple topological quasiparticles as information carriers in future spintronics nanocomputing.

\section{ACKNOWLEDGMENTS}

R.C. and Y.L. wish to acknowledge the China Scholarship Council (CSC) and the Department of Computer Science Kilburn Scholarship for the funding support. R.C. would like to thank the University of Manchester President's Scholarship. The authors would also like to acknowledge the assistance given by Research IT and the use of the Computational Shared Facility at the University of Manchester.

\section{APPENDIX: MICROMAGNETIC SIMULATIONS OF THE PROPOSED SKYRMIONIC INTERCONNECT}

The micromagnetic simulations were performed using the GPU-accelerated micromagnetic program MUMAX ${ }^{3}$ [63]. The time-dependent magnetization dynamics are conducted by the Landau-Lifshitz-Gilbert (LLG) equation,

$$
\frac{d \mathbf{m}}{d t}=-\left|\gamma_{\mathrm{LL}}\right| \mathbf{m} \times \mathbf{h}_{\mathrm{eff}}+\alpha \mathbf{m} \times \frac{d \mathbf{m}}{d t}+\frac{u}{t} \mathbf{m} \times\left(\mathbf{m}_{\mathrm{p}} \times \mathbf{m}\right),
$$

where $\mathbf{m}=\mathbf{M} / M_{S}$ is the reduced magnetization, $M_{s}$ is the saturation magnetization, $\gamma_{\mathrm{LL}}$ is the gyromagnetic ratio, $\mathbf{h}_{\mathrm{eff}}=$ $\mathbf{H}_{\text {eff }} / M_{s}$ is the reduced effective field, $\alpha$ is the damping parameter, $t$ is the thickness of the FM layer, and $\mathbf{m}_{\mathrm{p}}$ is the polarization direction. The energy density contains the Heisenberg exchange energy term, the anisotropy energy term, the Zeeman energy term, the magnetostatic energy term, and the interfacial DMI energy term. The input material parameters to perform the simulations are chosen following Ref. [41]: damping parameter, $\alpha=0.3$; DMI constant, $D_{\text {int }}=3.5 \mathrm{~mJ} \mathrm{~m}^{-2}$; the value for the Gilbert gyromagnetic ratio, $\gamma_{\mathrm{LL}}=-2.211 \times 10^{5} \mathrm{~mA}^{-1} \mathrm{~s}^{-1}$; saturation magnetization, $M_{s}=580 \mathrm{kA} \mathrm{m}^{-1}$; the spin Hall angle, $\Theta_{\mathrm{SH}}=0.6$, to enhance the spin Hall effect; the uniaxial out-of-plane magnetic anisotropy, $K_{u}=800 \mathrm{~kJ} \mathrm{~m}^{-3}$; and the exchange constant, which is assumed to be $A=15 \mathrm{pJ} \mathrm{m}^{-1}$. The size of the proposed pipelined skyrmionic interconnect is $2000 \mathrm{~nm}$ $\times 240 \mathrm{~nm} \times 1 \mathrm{~nm}$. In order to ensure the accuracy of calculation, the mesh size is set to $2 \mathrm{~nm} \times 2 \mathrm{~nm} \times 1 \mathrm{~nm}$, which is less than the exchange length $l_{\mathrm{ex}}=2 \sqrt{A /\left(\mu_{0} M_{s}^{2}\right)}=11.9 \mathrm{~nm}$.

For the simulation of the MML structure, we consider a tailored sandwich structure of $\left[\mathrm{HM}_{1}|\mathrm{FM}| \mathrm{HM}_{2}\right]_{n}$ and the material parameters are chosen according to the reported experimental results [42]: $\alpha=0.1, D_{\text {int }}=1.7 \mathrm{~mJ} \mathrm{~m}^{-2}, \gamma_{\mathrm{LL}}=$ $-2.211 \times 10^{5} \mathrm{~mA}^{-1} \mathrm{~s}^{-1}, M_{s}=956 \mathrm{kA} \mathrm{m}^{-1}, \Theta_{\mathrm{SH}}=0.6$, $K_{u}=717 \mathrm{~kJ} \mathrm{~m}^{-3}$, and $A=10 \mathrm{pJ} \mathrm{m}^{-1}$. An external magnetic field of $60 \mathrm{mT}$ in the out-of-plane direction is applied and the exchange length $l_{\mathrm{ex}}=2 \sqrt{A /\left(\mu_{0} M_{s}^{2}\right)}=5.9 \mathrm{~nm}$. In our multilayers, the intermediate $\mathrm{HM}_{1}$ and $\mathrm{HM}_{2}$ layers are thinner than the spin diffusion length. In this case, the torques would be efficient only in the external layers [64], so the spin orbit torque created via CPP is applied only in the first bottom and the first top layers and the injected spin polarization is uniform $(+y$ direction $)$ in these two layers.
[1] D. Suggs, M. Subramony, and D. Bouvier, The AMD "Zen 2" processor, IEEE Micro 40, 45 (2020).

[2] S. Borkar, Role of interconnects in the future of computing, J. Lightwave Technol. 31, 3927 (2013).

[3] P. Kogge and J. Shalf, Exascale computing trends: Adjusting to the "new normal" for computer architecture, Comput. Sci. Eng. 15, 16 (2013).

[4] M. Horowitz, Computing's energy problem (and what we can do about it), in 2014 IEEE International Solid-State Circuits Conference Digest of Technical Papers (ISSCC) (IEEE, New York, 2014), pp. 10-14.

[5] C. C. Liu, S. Chen, F. Kuo, H. Chen, E. Yeh, C. Hsieh, L. Huang, M. Chiu, J. Yeh, T. Lin et al., High-performance integrated fan-out wafer level packaging (InFO-WLP): Technology and system integration, 2012 International Electron Devices Meeting (IEEE, San Francisco, 2012), pp. 14.1.1-14.1.4.

[6] B. M. D. Sawyer, Y. Suzuki, R. Furuya, C. Nair, T.-C. Huang, V. Smet, K. Panayappan, V. Sundaram, and R. Tummala, Design and demonstration of a 2.5-D glass interposer BGA package for high bandwidth and low cost, IEEE Trans. Compon. Packag. Manuf. Technol. 7, 552 (2017).

[7] J. H. Lau, Heterogeneous Integrations (Springer, Berlin, 2019).
[8] J. Grollier, D. Querlioz, K. Y. Camsari, K. Everschor-Sitte, S. Fukami, and M. D. Stiles, Neuromorphic spintronics, Nat. Electron. 3, 360 (2020).

[9] Y. LeCun, Y. Bengio, and G. Hinton, Deep learning, Nature 521, 436 (2015).

[10] F. Jazaeri, A. Beckers, A. Tajalli, and J.-M. Sallese, A review on quantum computing: From qubits to front-end electronics and cryogenic MOSFET physics, in 2019 MIXDES: 26th International Conference on Mixed Design of Integrated Circuits and Systems (IEEE, New York, 2019), pp. 15-25.

[11] F. Arute, K. Arya, R. Babbush, D. Bacon, J. C. Bardin, R. Barends, R. Biswas, S. Boixo, F. G. S. L. Brandao, D. A. Buell et al., Quantum supremacy using a programmable superconducting processor, Nature 574, 505 (2019).

[12] Y. Huang, W. Kang, X. Zhang, Y. Zhou, and W. Zhao, Magnetic skyrmion-based synaptic devices, Nanotechnology 28, 08LT02 (2017).

[13] K. M. Song, J.-S. Jeong, B. Pan, X. Zhang, J. Xia, S. Cha, T.-E. Park, K. Kim, S. Finizio, J. Raabe et al., Skyrmion-based artificial synapses for neuromorphic computing, Nat. Electron. 3, 148 (2020).

[14] R. Chen, C. Li, Y. Li, J. J. Miles, G. Indiveri, S. Furber, V. F. Pavlidis, and C. Moutafis, Nanoscale Room-Temperature 
Multilayer Skyrmionic Synapse for Deep Spiking Neural Networks, Phys. Rev. Appl. 14, 014096 (2020).

[15] J. M. Hornibrook, J. I. Colless, I. D. Conway Lamb, S. J. Pauka, H. Lu, A. C. Gossard, J. D. Watson, G. C. Gardner, S. Fallahi, M. J. Manfra, and D. J. Reilly, Cryogenic Control Architecture for Large-Scale Quantum Computing, Phys. Rev. Appl. 3, 024010 (2015).

[16] A. Fert, V. Cros, and J. Sampaio, Skyrmions on the track, Nat. Nanotechnol. 8, 152 (2013).

[17] I. Dzyaloshinsky, A thermodynamic theory of "weak" ferromagnetism of antiferromagnetics, J. Phys. Chem. Solids 4, 241 (1958).

[18] X. Zhang, Y. Zhou, K. M. Song, T.-E. Park, J. Xia, M. Ezawa, X. Liu, W. Zhao, G. Zhao, and S. Woo, Skyrmion-electronics: Writing, deleting, reading and processing magnetic skyrmions toward spintronic applications, J. Phys.: Condens. Matter 32, 143001 (2020).

[19] K. Everschor-Sitte, J. Masell, R. M. Reeve, and M. Kläui, Perspective: Magnetic skyrmions-overview of recent progress in an active research field, J. Appl. Phys. 124, 240901 (2018).

[20] S. Rohart, J. Miltat, and A. Thiaville, Path to collapse for an isolated Néel skyrmion, Phys. Rev. B 93, 214412 (2016).

[21] C. Moutafis, S. Komineas, and J. A. C. Bland, Dynamics and switching processes for magnetic bubbles in nanoelements, Phys. Rev. B 79, 224429 (2009).

[22] J. Zang, M. Mostovoy, J. H. Han, and N. Nagaosa, Dynamics of Skyrmion Crystals in Metallic Thin Films, Phys. Rev. Lett. 107, 136804 (2011).

[23] N. Nagaosa and Y. Tokura, Topological properties and dynamics of magnetic skyrmions, Nat. Nanotechnol. 8, 899 (2013).

[24] X. Zhang, J. Xia, Y. Zhou, D. Wang, X. Liu, W. Zhao, and M. Ezawa, Control and manipulation of a magnetic skyrmionium in nanostructures, Phys. Rev. B 94, 094420 (2016).

[25] J. C. Slonczewski, A. P. Malozemoff, and O. Voegeli, Statics and dynamics of bubbles containing Bloch lines, in Magnetism and Magnetic Materials - 1972: Eighteenth Annual Conference, edited by H. C. Wolfe, C. D. Graham, and J. J. Rhyne, AIP Conf. Proc. No. 10 (AIP, New York, 1973), p. 458.

[26] A. P. Malozemoff and J. C. Slonczewski, Magnetic Domain Walls in Bubble Material (Academic Press, New York, 1979).

[27] S. Komineas and N. Papanicolaou, Topology and dynamics in ferromagnetic media, Physica D 99, 81 (1996).

[28] X. Zhang, G. P. Zhao, H. Fangohr, J. P. Liu, W. X. Xia, J. Xia, and F. J. Morvan, Skyrmion-skyrmion and skyrmion-edge repulsions in skyrmion-based racetrack memory, Sci. Rep. 5, 7643 (2015).

[29] Y. Zhang, S. Luo, B. Yan, J. Ou-Yang, X. Yang, S. Chen, B. Zhu, and L. You, Magnetic skyrmions without the skyrmion Hall effect in a magnetic nanotrack with perpendicular anisotropy, Nanoscale 9, 10212 (2017).

[30] B. Göbel, A. Mook, J. Henk, and I. Mertig, Overcoming the speed limit in skyrmion racetrack devices by suppressing the skyrmion Hall effect, Phys. Rev. B 99, 020405(R) (2019).

[31] X. Zhang, Y. Zhou, and M. Ezawa, Magnetic bilayer-skyrmions without skyrmion Hall effect, Nat. Commun. 7, 10293 (2016).

[32] M. Finazzi, M. Savoini, A. R. Khorsand, A. Tsukamoto, A. Itoh, L. Duò, A. Kirilyuk, T. Rasing, and M. Ezawa, Laser-Induced Magnetic Nanostructures with Tunable Topological Properties, Phys. Rev. Lett. 110, 177205 (2013).
[33] A. G. Kolesnikov, M. E. Stebliy, A. S. Samardak, and A. V. Ognev, Skyrmionium - high velocity without the skyrmion Hall effect, Sci. Rep. 8, 16966 (2018).

[34] C. Moutafis, S. Komineas, C. A. F. Vaz, J. A. C. Bland, T. Shima, T. Seki, and K. Takanashi, Magnetic bubbles in FePt nanodots with perpendicular anisotropy, Phys. Rev. B 76, 104426 (2007).

[35] A. Bogdanov and A. Hubert, The stability of vortex-like structures in uniaxial ferromagnets, J. Magn. Magn. Mater. 195, 182 (1999).

[36] C. Back, V. Cros, H. Ebert, K. Everschor-Sitte, A. Fert, M. Garst, T. Ma, S. Mankovsky, T. Monchesky, M. V. Mostovoy et al., The 2020 skyrmionics roadmap, J. Phys. D 53, 363001 (2020).

[37] Z. Li, C. Luo, T. Lu, J. Xu, W. Kong, and G. Guo, Cryogenic characterization and modeling of standard CMOS down to liquid helium temperature for quantum computing, arXiv:1811.11497.

[38] F. N. Rybakov and N. S. Kiselev, Chiral magnetic skyrmions with arbitrary topological charge, Phys. Rev. B 99, 064437 (2019).

[39] B. Jalali and S. Fathpour, Silicon photonics, J. Lightwave Technol. 24, 4600 (2006).

[40] D. C. Wong, G. De Micheli, M. J. Flynn, and R. E. Huston, A bipolar population counter using wave pipelining to achieve 2.5* normal clock frequency, IEEE J. Solid-State Circuits 27, 745 (1992).

[41] B. Göbel, A. F. Schäffer, J. Berakdar, I. Mertig, and S. S. P. Parkin, Electrical writing, deleting, reading, and moving of magnetic skyrmioniums in a racetrack device, Sci. Rep. 9, 12119 (2019).

[42] C. Moreau-Luchaire, C. Moutafis, N. Reyren, J. Sampaio, C. A. F. Vaz, N. Van Horne, K. Bouzehouane, K. Garcia, C. Deranlot, P. Warnicke et al., Additive interfacial chiral interaction in multilayers for stabilization of small individual skyrmions at room temperature, Nat. Nanotechnol. 11, 444 (2016).

[43] R. B. J. Bates and M. Bates, Principles of Voice \& Data Communications (McGraw-Hill, New York, 2006).

[44] W. J. Dally, W. J. Dally, and J. W. Poulton, Digital Systems Engineering (Cambridge University Press, Cambridge, UK, 1998).

[45] J. Sampaio, V. Cros, S. Rohart, A. Thiaville, and A. Fert, Nucleation, stability and current-induced motion of isolated magnetic skyrmions in nanostructures, Nat. Nanotechnol. 8, 839 (2013).

[46] R. Tomasello, E. Martinez, R. Zivieri, L. Torres, M. Carpentieri, and G. Finocchio, A strategy for the design of skyrmion racetrack memories, Sci. Rep. 4, 6784 (2014).

[47] L. Thomas, G. Jan, J. Zhu, H. Liu, Y.-J. Lee, S. Le, R.-Y. Tong, K. Pi, Y.-J. Wang, D. Shen et al., Perpendicular spin transfer torque magnetic random access memories with high spin torque efficiency and thermal stability for embedded applications, J. Appl. Phys. 115, 172615 (2014).

[48] X. Zhang, W. Cai, X. Zhang, Z. Wang, Z. Li, Y. Zhang, K. Cao, N. Lei, W. Kang, Y. Zhang et al., Skyrmions in magnetic tunnel junctions, ACS Appl. Mater. Interfaces 10, 16887 (2018).

[49] M.-H. Nguyen, D. C. Ralph, and R. A. Buhrman, Spin Torque Study of the Spin Hall Conductivity and Spin Diffusion Length in Platinum Thin Films with Varying Resistivity, Phys. Rev. Lett. 116, 126601 (2016). 
[50] Y. Cao, T. Sato, M. Orshansky, D. Sylvester, and C. Hu, New paradigm of predictive MOSFET and interconnect modeling for early circuit simulation, in Proceedings of the IEEE 2000 Custom Integrated Circuits Conference (IEEE, New York, 2000), pp. 201-204.

[51] W. F. Brown, Jr., Thermal fluctuations of a single-domain particle, Phys. Rev. 130, 1677 (1963).

[52] S. Buhrandt and L. Fritz, Skyrmion lattice phase in threedimensional chiral magnets from Monte Carlo simulations, Phys. Rev. B 88, 195137 (2013).

[53] B. D. Gates, Q. Xu, M. Stewart, D. Ryan, C. G. Willson, and G. M. Whitesides, New approaches to nanofabrication: Molding, printing, and other techniques, Chem. Rev. 105, 1171 (2005).

[54] D. Foster, C. Kind, P. J. Ackerman, J.-S. B. Tai, M. R. Dennis, and I. I. Smalyukh, Two-dimensional skyrmion bags in liquid crystals and ferromagnets, Nat. Phys. 15, 655 (2019).

[55] L. Camosi, N. Rougemaille, O. Fruchart, J. Vogel, and S. Rohart, Micromagnetics of antiskyrmions in ultrathin films, Phys. Rev. B 97, 134404 (2018).

[56] M. Hoffmann, B. Zimmermann, G. P. Müller, D. Schürhoff, N. S. Kiselev, C. Melcher, and S. Blügel, Antiskyrmions stabilized at interfaces by anisotropic Dzyaloshinskii-Moriya interactions, Nat. Commun. 8, 308 (2017).

[57] X. Zhang, J. Xia, Y. Zhou, X. Liu, H. Zhang, and M. Ezawa, Skyrmion dynamics in a frustrated ferromagnetic film and current-induced helicity locking-unlocking transition, Nat. Commun. 8, 1717 (2017).

[58] A. O. Leonov and M. Mostovoy, Edge states and skyrmion dynamics in nanostripes of frustrated magnets, Nat. Commun. 8, 14394 (2017).

[59] R. Dittrich, T. Schrefl, D. Suess, W. Scholz, H. Forster, and J. Fidler, A path method for finding energy barriers and minimum energy paths in complex micromagnetic systems, J. Magn. Magn. Mater. 250, 12 (2002).

[60] D. Cortés-Ortuño, W. Wang, M. Beg, R. A. Pepper, M.-A Bisotti, R. Carey, M. Vousden, T. Kluyver, O. Hovorka, and H. Fangohr, Thermal stability and topological protection of skyrmions in nanotracks, Sci. Rep. 7, 4060 (2017).

[61] L. Desplat, C. Vogler, J.-V. Kim, R. L. Stamps, and D. Suess, Path sampling for lifetimes of metastable magnetic skyrmions and direct comparison with Kramers' method, Phys. Rev. B 101, 060403(R) (2020).

[62] L. Desplat and J.-V. Kim, Entropy-Reduced Retention Times in Magnetic Memory Elements: A Case of the Meyer-Neldel Compensation Rule, Phys. Rev. Lett. 125, 107201 (2020).

[63] A. Vansteenkiste, J. Leliaert, M. Dvornik, M. Helsen, F. GarciaSanchez, and B. Van Waeyenberge, The design and verification of MuMax3, AIP Adv. 4, 107133 (2014).

[64] W. Legrand, J.-Y. Chauleau, D. Maccariello, N. Reyren, S. Collin, K. Bouzehouane, N. Jaouen, V. Cros, and A. Fert, Hybrid chiral domain walls and skyrmions in magnetic multilayers, Sci. Adv. 4, eaat0415 (2018). 